\title{
Educational Results of the Personal Exploration Rover Museum Exhibit ${ }^{*}$
}

\author{
Illah Nourbakhsh, Emily Hamner, and Brian \\ Dunlavey \\ The Robotics Institute \\ Carnegie Mellon University \\ Pittsburgh, PA, USA
}

\author{
Debra Bernstein and Kevin Crowley \\ Learning Research and Development Center \\ University of Pittsburgh \\ Pittsburgh, PA, USA
}

\begin{abstract}
The Personal Rover Project produces technology, curriculum and evaluation techniques for robotic educational use in formal and informal (after-school, out-ofschool) learning environments. Our specific aim for this phase of the project is to create and evaluate human-robot interactions that educate members of the general public in an informal learning environment, specifically museums. Our educational goals are to further an appreciation and understanding of NASA's Mars Exploration Rovers (MERs), to illustrate the role of robotic rovers in scientific exploration, and to provide hands-on learning experiences that demonstrate robot autonomy. We have designed a new robot, the Personal Exploration Rover (PER) and the related interactive components of a museum exhibit to achieve these goals. Here we describe the exhibits developed and the formal evaluation results of the exhibits' educational impact and efficacy. These results suggest techniques by which learning can be measured and used as an indicator of successful human-robot interaction.
\end{abstract}

Index Terms - Educational Robotics, Human-Robot Interaction, Social Robots, Robot Autonomy

\section{INTRODUCTION}

The need for significant improvement in technology literacy and education cannot be overstated, especially as technology becomes increasingly present in day-to-day human activities. Yet, in recent years, computer science and engineering departments have begun to suffer from declining student enrollment. We and other researchers have begun to explore the role that robotics can play in engaging and retaining students in technology-related curriculum and fields [2], [7], [12], [15], [19], [21]. This agenda has been furthered by our and others' results which show that educational robotics can trigger significant learning across broad educational themes that extend well beyond STEM (science, technology, engineering and mathematics) and into the associated lifelong learning skills of problem-solving, collaboration and communication [6], [9], [11], [16], [20], [22].

The Personal Rover Project, a multi-year educational robotics study, has focused specifically on the application of interactive, physically embodied robotic technology for education in formal and informal learning environments [8]. The educational goals of the project as a whole are:

- Inspire students to explore boundaries of their knowledge and creativity through the use of science and

\footnotetext{
* This work was funded by NASA/Ames and Intel Corporation.
}

technology and to pursue careers in math, science and engineering.

- Stimulate public awareness and interest in the NASA mission and reveal the challenges associated with using robotic devices for science and exploration.

- Teach children the critical skills of teamwork, collaboration, problem-solving and inquiry-based science.

Prior stages of the Personal Rover Project have identified design principles for the creation of richly expressive low-cost robotic platforms [10], and have deployed educational robotics curriculum for structured, formal learning environments [16]. Results derived from a formal analysis of a robotic autonomy summer course for high-school students included significant improvement in the sought-after area of retention of girls in technologyintensive coursework.

This paper describes the most recent endeavor of the Personal Rover Project, the creation and evaluation of a robot-based exhibit in informal learning environments that features unmediated, short-term human-robot interactions. In this phase of the project we focused on learning in informal settings where total time on task between visitors and robotic technologies is measured in minutes rather than days. Our goal was to assess whether robotic devices could offer significant educational advantages even in transient human-robot interactions, such as those experienced in a museum visit by tens of millions of visitors per year.

Motivated by the expected broad exposure and public interest in NASA's Mars Exploration Rover (MER) missions targeted to land in January 2004, we elected to launch a technology-based educational experience related to the MER missions in a number of science museums and technology centers across the country. Visitors would interact with the Personal Exploration Rover (PER), a robotic science rover (Fig. 1) via a kiosk-based 'mission control' interface to identify and search Martian rocks for signs of organic life.

The PER robot was designed to meet its specific educational objectives within the context of the NASA MER missions. Two key objectives were:

- Show that rovers are tools for doing science by enabling visitors to act as mission scientists, and use the PER robot to conduct a science operation.

- Enable visitors to appreciate the role of autonomy on board rovers. 


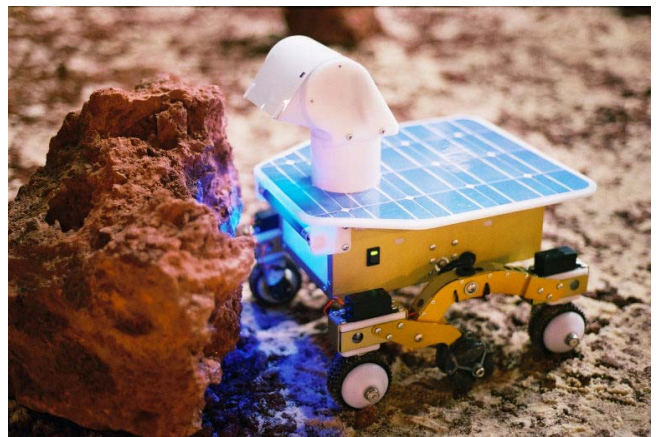

Fig. 1. A PER tests a rock for life at the National Science Center.

Museums are prime venues to evaluate these objectives because they offer human-robot interaction opportunities over a sufficiently large body of diverse visitors such that statistically meaningful conclusions regarding interaction and education can be drawn.

In just the first four months, PER robots effected more than 20,000 autonomous science target approaches and completed greater than 30 miles of rover travel with minimal operating failures. A detailed description of the PER robot can be found at [17] and [18].

\section{EXHIBIT INTERACTION}

The PER exhibit installations present museum visitors with the challenge of using the PER to search for signs of life on rocks placed in a Martian terrain sandbox called the "Mars yard". A PER mission starts with the rover compiling a $360^{\circ}$ panoramic image of the surrounding yard. At the 'mission control' kiosk located outside the Mars yard, users are presented with the panorama in the "Mission Builder" screen (Fig. 2) that guides them through the planning of a rover mission.

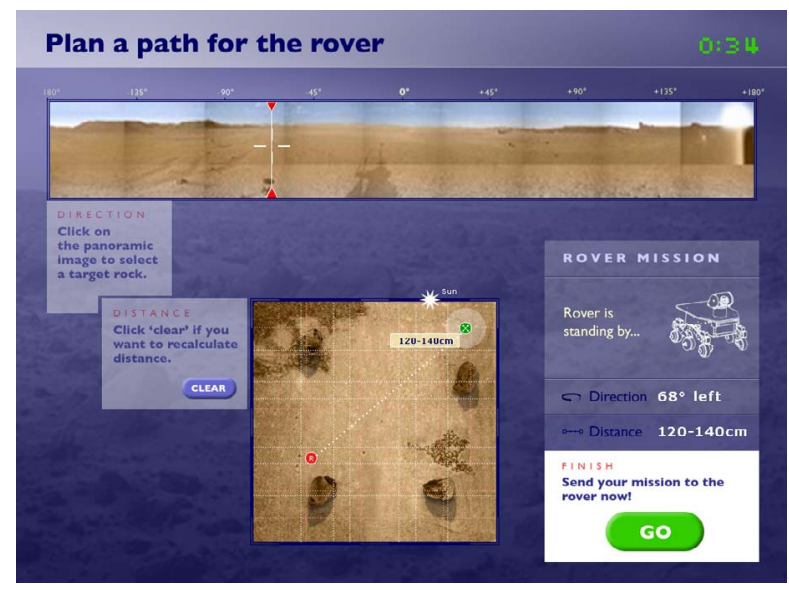

Fig. 2. The "Mission Builder" screen display.

First, users must interpret the panoramic image data and select which target rock to send the rover towards to search for signs of life. This first selection provides the rover orientation information for the mission. Second, users must locate the position of the rover and the selected target rock on an orthographic, overhead satellite map image. Together these provide sufficient rover angle and distance information to complete the mission. To help the user orient between the physical Mars yard and the onscreen panoramic display of the yard, a Martian sun is painted on the far wall of the yard, visible both from the kiosk and on the screen (Fig. 3). In addition, the rock positions, rock shapes, and the shape of the yard help users interpret the satellite map [17].

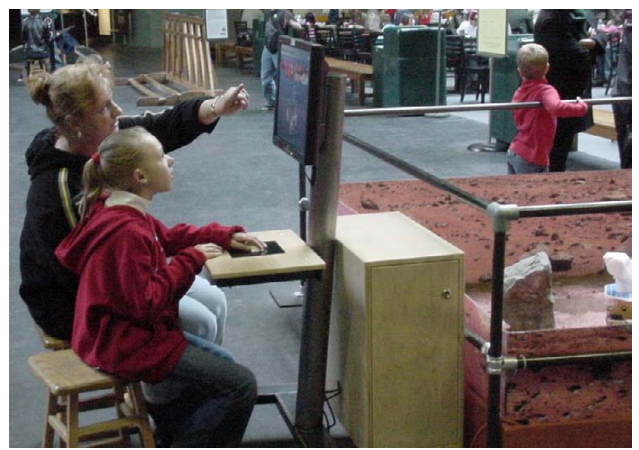

Fig. 3. The ability to see the yard and kiosk screen simultaneously aids users in orienting themselves within the exhibit.

As the rover executes the mission, a rover's-eye view camera allows visitors to experience the mission from the rover's perspective via real-time video. The "Rover Mission" sub-window at the bottom right of the "Mission Builder" screen remains visible during execution of the mission, providing data regarding rover operations, distance traveled and angles turned. Along the way the rover continually scans for obstacles in its path using an IR rangefinder mounted in its pan-tilt head. After the rover has turned and driven the distances specified, it demonstrates further autonomous capabilities by scanning for the target with the same IR rangefinder, determining if a target can be located, refining its position and alignment with the target rock, and performing an ultraviolet test for signs of life (Fig. 1). Some of the target rock faces are painted with an invisible fluorescing paint that glows under ultraviolet light. An image of the illuminated target rock is returned to the kiosk for scientific analysis by the user. The entire interaction is designed to be completed easily within three minutes to satisfy throughput requirements for high-traffic museums. An end message and countdown screen signal mission completion and allow the next user to step in.

\section{MUSEUM INSTALLATIONS}

The PER exhibit to date has been deployed at five main locations across the country: the Smithsonian Air and Space Museum (NASM), the Smithsonian Udvar-Hazy Center, the San Francisco Exploratorium, the National Science Center, and the NASA Ames Mars Center. The exhibit installations opened between December 29, 2003 and January 24, 2004, to coincide with the landing of the MERs on Mars, and each ran for two months or more. As of January 2005 the Udvar-Hazy and NASA Ames exhibits continue to operate and the exhibit is scheduled to open at the Japan World Expo in March 2005. 


\section{A. Exhibit format}

The presentation format for the exhibit is left up to the individual museum. As a result we have observed three different styles of exhibit interaction-mediated, semimediated and unmediated. At NASM a docent is stationed next to the 'mission control' kiosk, in order to provide information about the PER (and MER mission) and to guide the visitors through a mission. At the Hazy Center, the exhibit is used for structured teaching activities with school groups. We consider these types of interaction mediated as a human assists the user in the human-robot interaction. At the Exploratorium, National Science Center, and NASA Ames families interact with the PER robot exhibit on their own in an unmediated fashion, although staff members are generally available if needed to answer visitor questions.

\section{B. Mars yard designs}

Each museum designed and produced its own Mars yard or yards for the exhibit. The Mars yard terrain and topography are specifically designed with the PER's physical capabilities and the desired exhibit interaction in mind. The rocks, rubble and hills in the yard are all traversable by the rover except for four or five large rocks that serve as scientific targets. The yards are encircled by low walls portraying real Martian landscape and horizon imagery from NASA's Pathfinder mission. Each yard also features a sun on one wall designed to help the visitors orient themselves when using the exhibit. The hip walls are of sufficient height to be viewed as obstacles by the rover but low enough to allow visitors a view of the yard and rover.

The National Science Center and Exploratorium each have two yards, while the other locations each have a single yard. The dimensions and shapes of the Mars yards vary based on the space and material constraints of each location. The largest yard is at the NASA Ames Mars Center and measures 16 feet on each side. The smallest yard is approximately 8 feet by 9 feet. At the National Science center, the yards are polygons designed to maximize available space. The yards are constructed from spray painted Styrofoam; layered paint, glue, sand, wood and plaster; small lava rocks and sand; and layered Styrofoam, polymesh and dryvit compound.

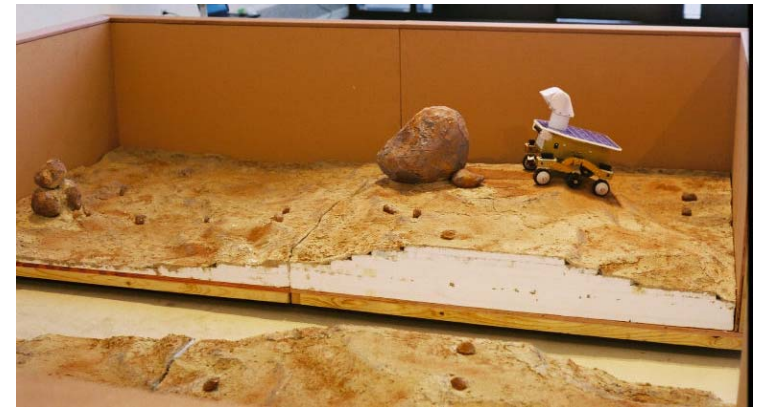

Fig. 4. This picture of the NASM yard was taken during installation of the exhibit, before the horizon images were added. The yard is built on casters and designed to split into quarters so that it can be easily moved.
Local high school students built both the NASM and Hazy Center yards. Using data from the Pathfinder mission, Earth Science classes designed the yard topography from cut Styrofoam to be an exact scale model of real Martian terrain. Art classes covered the foam with dryvit, painted the yard, and built realistic looking Styrofoam rocks. The end result is a realistic Martian terrain for the PERs to explore (Fig. 4).

\section{EXHIBIT USE PATTERNS}

Quantitative statistics regarding exhibit use were collected automatically at installations by the exhibit software itself and by sampled passive observation. Both quantitative results and informal observations guided the more formal educational exhibit evaluation that followed. These statistics identify the demographics of the exhibit users and the manner in which the exhibit was used. Significantly, the statistics show that time on task is extremely close to the design target of 3 minutes and more importantly virtually all exhibit users were able to successfully complete the entire mission. Together these statistics indicate that the distribution of time on task is not, as is often the case in museum exhibits, exponential but rather unimodal and narrow. Users who are engaged by the PER exhibit remain engaged through mission completion, then helpfully release control to the next museum visitor in queue. Details of both user demographics and mission use statistics follow.

\section{A. Audience}

Exhibit use observations were conducted at the Exploratorium and NASM. At both locations, the exhibit was in nearly constant use. Over roughly 4.5 hours of observation, 184 people interacted with the exhibit. This included 71 adult users (36 females and 35 males), and 113 child users ( 28 females and 85 males). The majority of exhibit users were in groups, and the average group size was $3.06(\sigma 1.22)$, with a total of 64 groups using the exhibit during this period. Group members often took turns conducting rover missions. Although more boys than girls were present at the exhibit, $61 \%$ of boys and $71 \%$ of girls attending the exhibit operated the rover.

\section{B. Mission statistics}

Based on logs automatically generated by the Exploratorium and NASA Ames kiosks between Dec $29^{\text {th }}$, 2003 and April 14 $4^{\text {th }}, 2004$ we are able to report additional information about exhibit use ${ }^{1}$. The exhibits were in use $75.4 \%$ of the time while they were open (331 hours idle and 1017 hours in use). Out of 26,200 missions only 525 $(2.0 \%)$ timed out before the end of the Mission Builder screen, meaning that $98 \%$ of users were able to successfully design a mission and send it to the rover. This represents a surprising retention statistic, in that users tend to engage and stay with the PER through an entire mission, virtually never leaving early. When a mission is

\footnotetext{
${ }^{1}$ All of the kiosks generate logs, but these results are based upon NASA/Ames and Exploratorium analyses only.
} 
unsuccessful, users are given the option to try again or quit. Only $499(1.9 \%)$ missions timed out at this stage, showing that users were highly engaged even when their mission failed to find the target rock. The average mission length was approximately 2 minutes 20 seconds (139.7 seconds $\sigma 60.1$ seconds). This is the length of time for a single set of instructions to be selected by the user, sent to the rover, and executed. On average each user engaged the PER in 1.6 missions ( $\sigma 0.94$ ), thus the overall individual time on task is approximately 4 minutes, exceeding the 1.4 minute engagement time typically seen at interactive science exhibits [5].

About half of the missions (52.7\%) ended with the rover successfully locating a rock (Fig. 5). The next most common outcome $(23.1 \%)$ was that the rover was blocked by an obstacle, generally a rock, while it still had more than $150 \mathrm{~cm}$ left in the path to its target. The rover went "out of range", i.e. detected a hip wall blocking its path, only $18.1 \%$ of the time. In $3.4 \%$ of the missions, the mission ended due to a robot error such as failed communication. Because the IR sensor's range is limited to $150 \mathrm{~cm}$, the rover was unable to locate any rock or hip wall $2.7 \%$ of the time.

In summary it is clear both from time on task values, time-out rarity and mission success rates that visitors are able to effectively use the PER exhibit, even in the unmediated cases of the Exploratorium and NASA/Ames installations. It is further clear that for children, there is no obvious statistical gender gap in terms of engagement with the PER exhibit. Both of the above conclusions are hopeful in that the PER exhibit attracts and engages the target population. The ability of the PER exhibit to engage and retain the interest of girls as well as boys is noteworthy; this echoes earlier educational results from the Robotic Autonomy summer course [16]. The next question, addressed in the following section, is whether this exhibit uses technology in an educational manner.

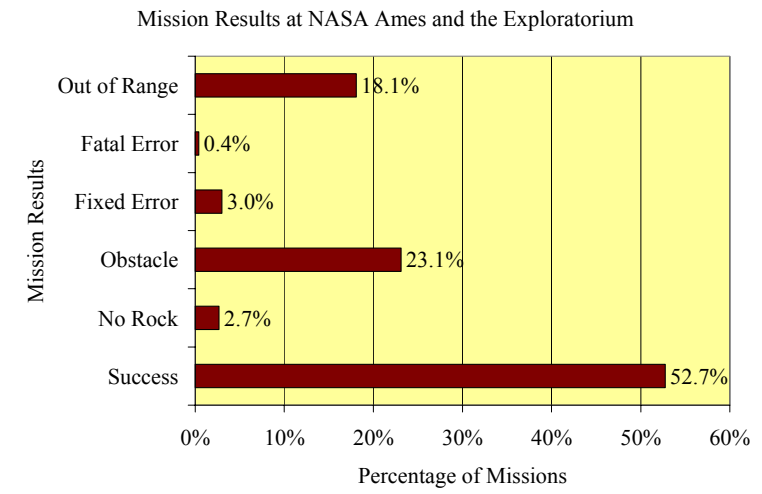

Fig. 5. Mission results from NASA Ames and the Exploratorium between December $29^{\text {th }}, 2003$ and April 14 ${ }^{\text {th }}, 2004$.

\section{EXHIBIT ANALYSIS}

The Learning Research \& Development Center conducted formal educational evaluation of the PER exhibit at NASM and the Exploratorium. These two museums were chosen as research sites in order to provide a full picture of how the exhibit functioned with different levels of museum mediation. The goal of the evaluation was to see if people were engaging with the intended content of the exhibit.

Traditional school-based assessments of learning are often inappropriate for use in informal learning settings [1]. As groups of visitors use and talk about exhibits, they are constructing a shared understanding of the content. Following recent theoretical and empirical work in museum learning [4], [14], our analyses focus on this naturally occurring talk as the best indicator of whether the exhibit successfully meets its educational goals.

In this article, we focus upon one of the most common exhibit user groups: children visiting the museum with families. We first analyze videotapes of families using the exhibit in order to describe the extent to which their conversations reflect the intended educational themes. Second, we analyze post-exhibit interviews with children in order to describe the extent to which they understood those same themes after having used the exhibit.

The post-exhibit child interview consisted of a set of open-ended questions about the Mars mission, the MERs, and the PERs. Here we present selected analyses focusing on the questions of how the exhibit supported the two educational objectives of allowing visitors to explore 1) the role of robots in mission science and 2) the nature of robot autonomy. For additional analyses see [17].

\section{A. The Role of Robots in Mission Science}

In this section, we evaluate the extent to which the exhibit supported visitor learning of the role of robots in scientific exploration. Fig. 6 presents the percentage of conversational groups ${ }^{2}$ discussing different topics. These data suggest that the PER exhibit supported conversations about the Mars mission and general robotics at both sites. However, conversation groups at NASM, which included a docent, were significantly more likely to talk about the Mars mission and to make explicit comparisons between the MER and the PER.

Further analysis of the conversation data revealed that parents generally initiated the same amount of thematic talk at both the Exploratorium and NASM exhibits ${ }^{3}$, and that the docents seem to be responsible for the increase in the amount of thematic talk at NASM. However, even if docents are able to provide additional factual information to museum visitors, is it not necessarily the case that the PER exhibit is more successful when mediated by a docent. Anecdotal evidence suggests that parents are more sensitive to their children's understanding of the rover, and are more likely to tailor their comments at the exhibit

\footnotetext{
${ }^{2}$ As a unit of analysis, the conversational group includes anyone present at the exhibit with the child. At the Exploratorium, the conversational group generally included the child, parent(s), siblings and any other exhibit users with whom the child interacted. At NASM, the conversational group included the child, parent(s), siblings, other exhibit users, and a docent.

${ }^{3}$ With the exception of talk about collaboration with robots (i.e., people and robots working together to solve problems), which was initiated more often by parents at the Exploratorium.
} 
to correct children's misperceptions. Research in the field of museum learning suggests that parents can serve an important bridging function between children and museum exhibits, making the parent's role an important one [3].

When interviewed after exhibit use, almost all children at both the Exploratorium and NASM possessed basic knowledge of the Mars rover mission (93\% and 100\% respectively). Additionally, $21 \%$ of children at the Exploratorium and $38 \%$ of children from NASM made spontaneous comparisons between the MER and the PER.

With regard to person-robot communication, $72 \%$ of children at the Exploratorium and $69 \%$ of children at NASM were able to describe the devices people can use to communicate with robots (e.g., computers and, in the case of rovers in space, satellites). There were no statistically significant differences between children from NASM and the Exploratorium for any of the categories reported here.

\begin{tabular}{|l|l|l|}
\hline Themes & Exploratorium & NASM \\
\hline About the Mars Mission* & $55 \%$ & $93 \%$ \\
\hline Comparisons between MER and PER* & $24 \%$ & $79 \%$ \\
\hline Communicating with Robots & $45 \%$ & $72 \%$ \\
\hline Collaborating with Robots & $86 \%$ & $93 \%$ \\
\hline
\end{tabular}

*indicates a statistically significant difference between the Exploratorium and NASM groups, $\mathrm{p}<.01$

Fig. 6. Percentages of conversation groups at each museum discussing themes related to the role of robots in mission science.

\section{B. The Nature of Robot Autonomy}

This exhibit was designed to provide museum visitors with the knowledge and information necessary to appreciate the importance of rover autonomy. Although all museum visitors come to the exhibit with prior ideas of what robots are and what they can do, most have probably not interacted with a robot that possessed true autonomous properties [13]. Thus, the exhibit experience provides a unique opportunity for visitors to re-evaluate their concepts of what a robot is and what a robot is capable of doing.

Fig. 7 shows the percentage of conversational groups discussing different topics at the Exploratorium and NASM. Conversational groups at both museums addressed each topic, although all topics were addressed significantly more frequently at NASM. Analysis of the source of exhibit conversation revealed that parents at both the Exploratorium and NASM discussed these topics with similar frequency. As was the case in the previous set of exhibit conversation analyses, the docents seem to be responsible for the increase in frequency of thematic talk at NASM.

\begin{tabular}{|l|l|l|}
\hline Themes & Exploratorium & NASM \\
\hline Rover Design* & $34 \%$ & $93 \%$ \\
\hline Rover Activities* & $45 \%$ & $100 \%$ \\
\hline Rover Autonomy* & $52 \%$ & $93 \%$ \\
\hline
\end{tabular}

*indicates a statistically significant difference between the Exploratorium and NASM groups, $\mathrm{p}<.01$

Fig. 7. Percentage of conversation groups at each museum discussing themes related to rover autonomy.
In order to assess children's ideas about rover capabilities, children's interview transcripts were coded using two categories: rover design and rover activities. Children from both the Exploratorium and NASM were able to speak knowledgably about the technology on the rovers and the type of actions they were capable of performing. Fifty-two percent of children from the Exploratorium and $77 \%$ of children from NASM talked about rover design (e.g., the technology typically found in rovers, such as motors, cameras, range finders). Similarly, $55 \%$ of children at the Exploratorium and $85 \%$ of children at NASM were able to describe the types of activities a rover could perform (e.g., taking pictures, driving, exploring); this difference was marginally significant, $\mathrm{X}^{2}$ $(1, N=42)=3.39, \mathrm{p}=.06$.

Assessing children's ideas about rover autonomy proved to be more challenging, as some children were inconsistent or unsure of whether a robot would be capable of autonomous behavior. To address this issue, we devised a separate system to measure both the adequacy and the strength (consistency) of children's ideas about robotic autonomy. For each statement indicating an understanding of the autonomous operations of the rover, children were given one positive point. For each statement indicating the opposite belief, namely that the rovers were incapable of independent action and operated via remote control, children were given one negative point. This system was applied to children's answers to open-ended questions about how the rovers operate.

Neither PER nor MER autonomy scores correlated significantly with age, although, as one might expect, PER and MER scores were significantly correlated with each other $(\mathrm{r}=0.48, \mathrm{n}=42, \mathrm{p}=.001)$. Across institutions, there were no significant differences in PER or MER autonomy scores.

In total, over $40 \%$ of children at the Exploratorium and over $45 \%$ of children at NASM left the exhibit with some understanding of the autonomous capabilities of the PER. Similarly, 31\% of children at the Exploratorium and over $50 \%$ of children at NASM came away understanding the autonomous capabilities of the $\mathrm{MER}^{4}$. The somewhat higher autonomy scores at NASM may be a function of the explicit conversation from docents regarding robot autonomy. Cognizant of the goals of the exhibit, docents were more likely to be explicit in their descriptions of the rover's autonomous behavior than were parents. It may be the case that for a concept as difficult as robotic autonomy, children benefit from explicit descriptions and definitions of autonomous behavior.

\section{Analysis Conclusions}

This assessment suggests that the exhibit was successful in meeting its core goals of involving visitors in explorations of the role of robots in mission science and of robots as autonomous entities. Analysis of family conversation suggests that visitors were expanding on relevant themes as they used the exhibit. They talked

\footnotetext{
${ }^{4}$ These percentages represent the number of children with positive autonomy scores for the MER and PER.
} 
about the ongoing Mars mission, compared the MER and PER, discussed communicating and collaborating with robots, and talked about robot design, technology, and autonomy. Interviews with children following the exhibit suggested that almost all children were aware of the Mars mission and that many of them also were able to connect the exhibit experience in specific ways to the mission. Children did not end their experience with a uniformly robust view of autonomy. Although some recognized autonomous characteristics of the rovers, most children held inconsistent theories. More than half still held views that the rovers are primarily operated through direct remote-control. We do not necessarily believe that a single exhibit experience would be a sufficient base for children to develop fully correct theories of autonomy. The exhibit experience is probably best seen as a chance for families to work out some of these issues in the context of an authentic autonomous rover. Still, future versions of such exhibits should be designed to more explicitly challenge children's incorrect or inconsistent theories.

\section{CONCLUSIONS}

The Personal Exploration Rover has served as a rewarding demonstration of educational robotics applied to the informal learning space. Given concrete goals in relation to the NASA Mars Exploration Rover mission, this team designed a new educational rover and graphical interaction system, installed the resulting exhibit at multiple high-traffic museums across the country, and performed quantitative and qualitative evaluation of the exhibit's efficacy. In summary this project demonstrates that robotic technology has compelling value in the museum setting, and that concrete educational results can be achieved and measured in such a setting. Exhibit statistics suggest that, among children, girls and boys are both engaged by this robotic exhibit, to such a degree that virtually all users succeed in the completion of an entire scientific rover mission. Educational evaluation suggests that the exhibit effectively serves as a platform for family discussions about the MER mission and robotics, and that children come away from the exhibit with measurable knowledge in these areas. These results also indicate that learning can be evaluated and used as a critical measure of successful human-robot interactions.

As robotic technology advances, future teams will be capable of creating ever more compelling exhibits and curricula for both formal and informal learning venues. We hope that this project can serve as a motivation for future teams to not only research, dream and invent, but also to harden, fabricate and install so that thousands can benefit from these educational technology ventures.

\section{ACKNOWLEDGMENT}

We would like to thank Ellen Ayoob, Doug Baldwin, Jim Butler, Corinne Cannon, Daniel Clancy, Jeff Cross, Maylene Duenas, Joe Edwards, Edward Epp, Jim Frye, Rachel Gockley, Jean Harpley, Robyn Higdon, Thomas Hsiu, Mark Lotter, Marti Louw, Andrew McClellan, Nicole Minor, Victor Morales, Jennifer O'Brien, Anuja Parikh, Eric Porter, Mike Reeves, Skip Shelly, Kristen Stubbs, Tom Roach, Priscilla Strain, Nick
Veronico, Noel Wanner, Ollie Washington, Steven Williams, Peter Zhang, and Cheryl Zimmerman.

\section{REFERENCES}

[1] Allen, S. Looking for learning in visitor talk: A methodological exploration. In G. Leinhardt, K. Crowley \& K. Knutson (Eds.) Learning Conversations in Museums (pp. 259-303). Mahwah, NJ: Lawrence Erlbaum Associates (2002).

[2] Beer, R., Chiel, H., \& Drushel, R. Using autonomous robots to teach science and engineering. Communications of the $A C M$, June (1999).

[3] Crowley, K. \& Callanan, M. Describing and supporting collaborative scientific thinking in parent-child interactions. Journal of Museum Education, 23, 12-17 (1998).

[4] Crowley, K., Callanan, M., Jipson, J., Galco, J., Topping, K. \& Shrager, J. Shared scientific thinking in everyday parent-child activity. Science Education, 85(6), 712-732 (2001).

[5] Crowley, K., Callanan, M., Tenenbaum, H. \& Allen, E. Parents explain more often to boys than to girls during shared scientific thinking. Psychological Science, 12(3), 258-261 (2001).

[6] Druin, A. \& Hendler, J. Robots for kids: exploring new technologies for learning, The Morgan Kaufmann Series in Interactive Technologies, Morgan Kaufmann, (2000).

[7] Ebert-Uphoff, I. Introducing parallel manipulators through laboratory experiments. IEEE Robotics \& Automation Magazine, 10 (3), pp. 13-19, (2003).

[8] Falcone, E., Gockley, R., Porter, E. \& Nourbakhsh, I, The personal rover project, Special Issue on Socially Interactive Robots, Robotics and Autonomous Systems, (2003).

[9] Gerovich, O., Goldber, R. P., \& Donn, I. D. From science projects to the engineering bench. IEEE Robotics \& Automation Magazine, 10 (3), pp. 9-12, (2003).

[10] Hsiu, T., Richards, S., Bhave, A., Perez-Bergquist, A. \& Nourbakhsh, I. Designing a Low-cost, Expressive Educational Robot. In Proceedings of IROS 2003. Las Vegas, USA, (2003).

[11] Kitts, C. Surf, turf, and above the Earth. IEEE Robotics \& Automation Magazine, 10 (3), pp. 30-36, (2003).

[12] Kumar, D. \& Meeden, L. A robot laboratory for teaching artificial intelligence. In Proc. of $29^{\text {th }}$ SIGCSE Symposium on Computer Science Education, (1998).

[13] Leinhardt, G. \& Crowley, K. Objects of learning, objects of talk: Changing minds in museums. In S.G. Paris (Ed) Perspectives on Object-Centered Learning in Museums (pp. 301-324). Mahwah, NJ: Lawrence Erlbaum Associates (2002).

[14] Leinhardt, G., Crowley, K. \& Knutson, K. (Eds.). Learning Conversations in Museums. Mahwah, NJ: Lawrence Erlbaum Associates (2002)

[15] Murphy, R. Introduction to AI Robotics. MIT Press, (2000).

[16] Nourbakhsh, I., Crowley, K., Bhave, A., Hamner, E., Hsiu, T., Perez-Bergquist, A., Richards, S., \& Wilkonson, K. The Robotic Autonomy Mobile Robotics Course: Robot design, curriculum design and educational assessment. Autonomous Robotics Journal, in print. (2004).

[17] Nourbakhsh, I., Hamner, E., Bernstein, D., Crowley, K., Porter, E., Hsiu, T., Dunlavey, B., Ayoob, E., Lotter, M., Shelly, S., Parikh, A., \& Clancy, D. The Personal Exploration Rover: The Ground-up Design, Deployment and Educational Evaluation of an Educational Robot for Unmediated Informal Learning Sites. Carnegie Mellon University Technical Report CMU-RI-TR-04-38. August, (2004).

[18] Nourbakhsh, I., Hamner, E., Porter, E., Dunlavey, B., Ayoob, E., Hsiu, T., Lotter, M., \& Shelly, S. The Design of a Highly Reliable Robot for Unmediated Museum Interaction. In Proc. International Conference on Robotics and Automation. IEEE ICRA (2005).

[19] Nourbakhsh, I. When students meet robots. Essay in IEEE Intelligent Systems and Their Applications, 15(6), p15. (2000).

[20] Nourbakhsh, I. Robotics and education in the classroom and in the museum: On the study of robots, and robots for study. In Proceedings Workshop for Personal Robotics for Education. IEEE ICRA (2000).

[21] Papert, S. \& Harel, I. Situating Constructionism, in: Constructionism, Ablex Publishing Corp., (1991).

[22] Wolz, U. Teaching design and project management with Lego RCX robots. In Proc. SIGCSE Conference (2000). 